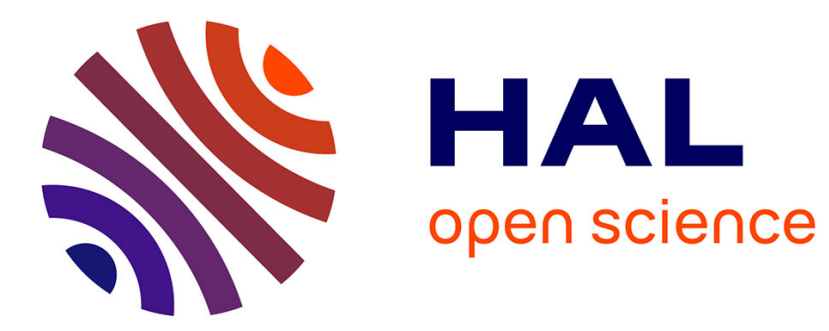

\title{
Torsional control of the methyl group in methanol
}

\author{
L. Coudert, F. Billard, E. Hertz, O. Faucher, B. Lavorel
}

\section{To cite this version:}

L. Coudert, F. Billard, E. Hertz, O. Faucher, B. Lavorel. Torsional control of the methyl group in methanol. Physical Review A, 2019, 100 (4), 10.1103/PhysRevA.100.043425 . hal-02367925

\section{HAL Id: hal-02367925 \\ https://hal.science/hal-02367925}

Submitted on 4 Sep 2020

HAL is a multi-disciplinary open access archive for the deposit and dissemination of scientific research documents, whether they are published or not. The documents may come from teaching and research institutions in France or abroad, or from public or private research centers.
L'archive ouverte pluridisciplinaire HAL, est destinée au dépôt et à la diffusion de documents scientifiques de niveau recherche, publiés ou non, émanant des établissements d'enseignement et de recherche français ou étrangers, des laboratoires publics ou privés. 


\title{
Torsional control of the methyl group in methanol
}

\author{
L. H. Coudert ${ }^{1},{ }^{*}$ F. Billard ${ }^{2}$, E. Hertz ${ }^{2}$, O. Faucher ${ }^{2}$, and B. Lavorel ${ }^{2}$ \\ ${ }^{1}$ Institut des Sciences Moléculaires d'Orsay (ISMO), CNRS, \\ Univ. Paris-Sud, Université Paris-Saclay, F-91405 Orsay, France and \\ ${ }^{2}$ Laboratoire Interdisciplinaire Carnot de Bourgogne (ICB), \\ UMR 6303 CNRS-Université Bourgogne-Franche Comté, \\ 9 Av. A. Savary, BP 47 870, F-21078 Dijon Cedex, France
}

(Dated: September 4, 2020)

\begin{abstract}
Experimental and theoretical investigations of the field-free alignment of the non-rigid methanol molecule are reported. The molecule is subject to a $140 \mathrm{TW} / \mathrm{cm}^{2}$ intensity laser pulse with a $100 \mathrm{fs}$ duration. The experimental signal displays a constant permanent alignment and a fast decaying transient alignment consistent with a prolate-like molecule with $(B+C) / 2$ on the order of $0.808 \mathrm{~cm}^{-1}$. The theoretical model takes into account the large amplitude internal rotation of the methyl group with respect to the hydroxyl group. In the case of a continuous wave laser field, a rotational alignment close to that of a rigid molecule is predicted. Torsional alignment also occurs even though there is no explicit dependence of the polarizability tensor on the angle of internal rotation. In the case of a strong short laser pulse, the theoretical approach shows that permanent and transient rotational alignment take place. The latter displays an exponential-like decay due to the high density of rotation-torsion levels. Torsional alignment also occurs and depends on the temperature. The theoretical model allows us to reproduce the experimental signal provided one component of the polarizability tensor is adjusted and dissipation effects due to molecular collisions are taken into account.
\end{abstract}

\section{INTRODUCTION}

Understanding the behavior of non-rigid molecules when subject to intense laser or terahertz pulses is an interesting field of investigation with many promising applications [1, 2]. This behavior was studied so far in non-rigid molecules consisting of two subunits which can rotate with respect to each other. Depending on the nature of these subunits, it is useful to distinguish two types of molecular systems. Highly symmetrical ethylene-like molecules [3] including biphenyl $\left(\mathrm{C}_{12} \mathrm{H}_{22}\right)$, its halogenated derivatives, and diboron tetrafluoride $\left(\mathrm{B}_{2} \mathrm{~F}_{4}\right)$ constitute the first type. In these molecules, the two subunits are identical, or nearly identical, and the polarizability tensor, strongly dependent on their respective orientation, leads to a large amplitude torsional motion strongly coupled to the electric field through quadratic Stark effect. For this reason the behavior of these prototypical molecules when subject to a strong short electric field was extensively studied both theoretically [4-11] and experimentally [12-14]. In the second type of molecules, the two subunits are different and the polarizability tensor can be approximated by that of the larger one. It is weakly dependent on the large amplitude coordinate which is not directly coupled to the electric field. The only molecular system of this type studied so far is the weakly bound Indole $-\mathrm{H}_{2} \mathrm{O}$ cluster consisting of a water molecule attached to an indole molecule [15]. The former undergoes a large amplitude internal rotation about its $b$-axis with the axis of internal rotation lying in the symmetry plane of the indole subunits. Despite the weak

* laurent.coudert@u-psud.fr dependence of the polarizability tensor on the angle of internal rotation, the torsion can still be controlled by the laser field [15].

The behavior of a methanol molecule subject to an intense laser pulse is investigated both experimentally and theoretically. Methanol obviously belongs to the second type of molecules as this non-rigid species consists of two different subunits, the hydroxyl and methyl groups, the latter undergoing internal rotation with respect to the former. The present paper reports the experimental alignment signal recorded after subjecting the molecule to a $100 \mathrm{fs}$ duration laser pulse with a $140 \mathrm{TW} / \mathrm{cm}^{2}$ intensity. The theoretical model developed allows us to evaluate the effects of the laser field treating simultaneously the overall rotation of the molecule and the large amplitude torsional motion. Unlike in the previous one- or two-dimensional models used in non-rigid molecules $[4,5,8,13,16,17]$, the present model accounts for all three rotational degrees of freedom. No assumptions about the molecule being already aligned or oriented prior to the laser pulse are made.

In the case of a continuous wave (c.w.) laser field, the theoretical model predicts a rotational alignment, qualitatively similar to that of a rigid asymmetric molecule, and a temperature dependent torsional alignment. When the methanol molecule is subject to the strong laser pulse, the theoretical treatment leads to a time dependent rotational alignment which resembles that of a rigid molecule within 50 ps from the pulse. Beyond this time, the behavior of the molecule departs from that of a rigid molecule as an exponential-like decay of the revival amplitude is calculated. The theoretical model allows us to reproduce fairly accurately the experimental signal provided collision induced dissipation effects are considered and a molecular parameter is adjusted. 
The paper has three remaining sections. In Section II the experimental setup is outlined and the experimental signal is qualitatively described. The theoretical approach used to model the effects of the laser field is introduced in Section III. Three sets of results are presented in Section IV including the effects of a static and time dependent laser field and the analysis of the experimental signal. Section V is the discussion.

\section{EXPERIMENTAL SETUP}

The experimental setup shown in Fig. 1 is used to measure the time-dependent birefringence of the molecular gas sample after excitation by a strong short laser pulse. The strong pump and weak probe pulses are both derived from a Chirp Pulse Amplifier (CPA, 796 nm, $1 \mathrm{kHz}$ repetition rate, $100 \mathrm{fs}$ pulse duration, $3 \mathrm{~mJ}$ ). The energy and polarization of the two beams are controlled by means of half-wave plates and polarizers. There are focused with a $100 \mathrm{~mm}$ focal lens and overlapped in a gas cell. The probe pulse is frequency doubled in a type I BBO crystal. The probe laser pulse, time delayed with respect to the pump one, is initially vertically polarized, whereas the pump polarization is set at $45^{\circ}$. The polarization state of the probe beam after interaction with the excited molecules is measured using a balanced detection. The detection device consists of a quarter wave-plate followed by a Wollaston prism and a balanced pair of photodiodes which measure independently the horizontal and vertical polarization components of the probe beam. The two photodiodes are connected head-to-tail so that the difference of their signals $S= \pm\left(S_{\mathrm{H}}-S_{\mathrm{V}}\right)$ is directly obtained, amplified, and sent to a lock-in amplifier synchronized with the laser repetition rate. The quarter-wave plate is oriented so as to get a circular polarization without pump pulse. One of the advantages of this detection scheme is the cancellation of the laser intensity fluctuations. The measured birefringence can be determined by a simple Jones-matrix analysis [18]:

$$
S_{\text {bir }}=P_{\|} P_{\perp} \varepsilon_{\mathrm{p}}^{2} \sin (\delta),
$$

where $P_{\|}\left(P_{\perp}\right)$ is the transmission coefficient of the electric field amplitude parallel (perpendicular) to the pump polarization direction, $\varepsilon_{\mathrm{p}}$ is the probe electric field amplitude, and $\delta$ is the phase difference between these two perpendicular directions. In the present case of non-resonant excitation, the transmission coefficients are close to 1 . If the phase difference is small enough, $S_{\text {bir }}$ is proportional to the birefringence, which is the difference of refractive index along the and perpendicular to the pump polarization: $S_{\text {bir }} \propto \Delta n=(\lambda / 2 \pi L) \delta$, where $L$ is the length of the sample or the interaction length. The $\mathrm{CH}_{3} \mathrm{OH}$ sample is initially stored in the liquid phase and vaporized by expansion into the gas cell under vacuum in order to reach a pressure of about 90 millibar at room temperature. The pump laser pulse is assumed to be Gaussian with a maximum intensity of $140 \mathrm{TW} / \mathrm{cm}^{2}$ and a half

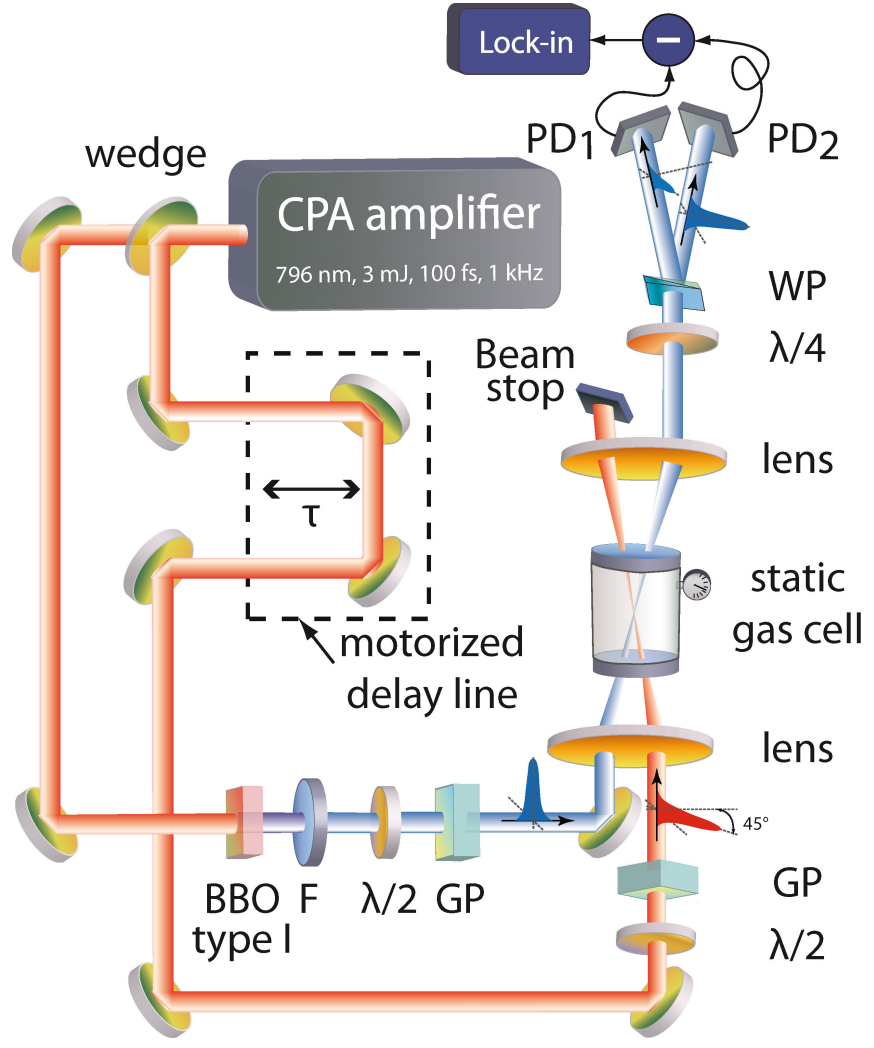

Figure 1. Experimental setup and the balanced detection scheme. F: $400 \mathrm{~nm}$ pass-band filter, $\lambda / 2$ : half-wave plate, $\lambda / 4$ : quarter-wave plate, GP: Glan polarizer, WP: wollaston prism, PD1, PD2: photodiodes.

width at half maximum (HWHM) of 50 fs. For comparison with the experimental data, the signal $S_{\text {bir }}$ has to be convolved with the probe pulse intensity. The crossing angle between the pump and the probe beams leads to a loss of temporal resolution which can be accounted for through a convolution with a Gaussian function of about 100 fs (HWHM) representing the probe pulse intensity.

Figure 2 shows the time evolution of the experimental signal. Five revivals with decreasing amplitude can clearly be seen in the field-free alignment signal as well as a permanent alignment of seemingly constant amplitude. The difference of behavior between permanent and transient alignment (i.e., revivals) with respect to collisional dissipation is well understood in linear and symmetric top molecule and was recently observed in the ethane molecule [19]. The explanation is based [20] on the propensity of inelastic collisions to preserve the orientation of molecules as defined by the ratio $M / J$, where $J$ is the total rotational angular momentum quantum number and $M$ its projection with respect to the space-fixed $Z$ axis. The consequence is that the permanent alignment decreases much more slowly than the revivals. Since in the prolate-like methanol molecule at low pressure we only observe a decrease of the transient alignment, only collisional dissipation of the revivals will be considered in 


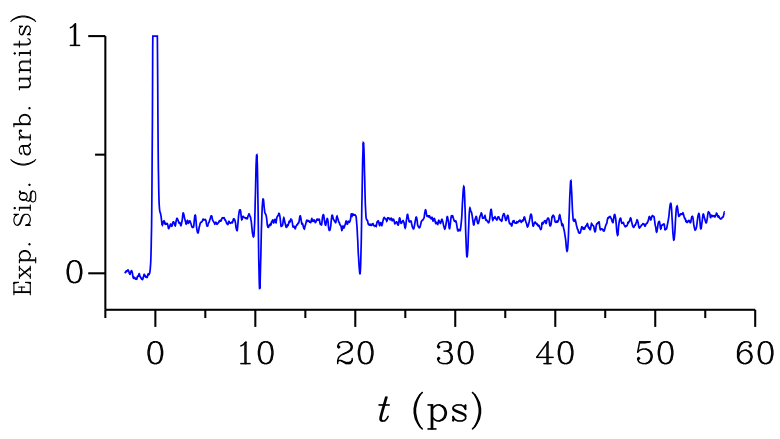

Figure 2. The experimental alignment signal recorded with the setup of Section II is plotted as a function of the time delay $t$ in picoseconds between the pump and probe laser pulses. For clarity, the strong signal near $t=0$ is truncated.

the theoretical model presented in the next section.

\section{THEORY}

Theoretical approaches designed to compute the rotation-torsion energy levels of methanol and methanollike molecules are already available and the one selected in this investigation is described below focusing on the way the molecular-fixed axis system is attached to the molecule and on symmetry considerations. The polarizability tensor, taken from a previous $a b$ initio investigation, is transformed to be compatible with the present axis system. The dissipation mechanism chosen is presented.

\section{A. Rotation-torsion Hamiltonian}

The coordinates [21,22] used in this work consist of the three usual Eulerian angles and of the torsional angle $\gamma$. As shown by Fig. 3, the latter parameterizes the internal rotation of the methyl group with respect to the frame containing the carbon atom and the hydroxyl group oxygen and hydrogen atoms. The molecule-fixed $x y z$ axis system is attached to the molecule so that its origin coincides with the molecular center of mass. The three atoms of the frame and the axis of internal rotation are held fixed in this axis system and lie in the $x z$ plane. Atom positions in the molecule-fixed axis system are illustrated in Fig. 3 where the three equivalent hydrogen atoms of the methyl group are numbered 1,2, and 3; the hydrogen atom of the hydroxyl group being atom 4. $\gamma=0$ is the staggered configuration with hydrogen atom 1 in the $x z$ plane and anti to hydrogen atom 4 . As stressed by Hougen et al. [23], the symmetry group to be used is $G_{6}$; its character table and the transformation properties of the coordinates are given in Tables VII and VIII of this reference.

Rotation-torsion energy levels are calculated with the the rho axis method (RAM) internal-rotation Hamilto-
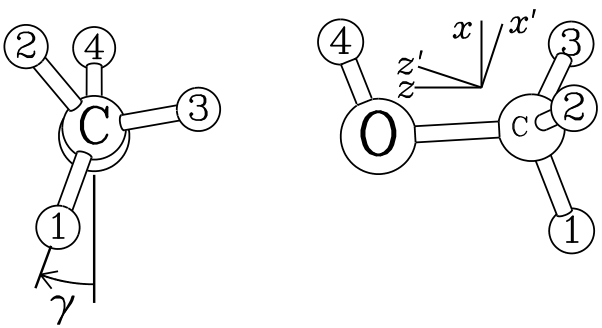

Figure 3. The left panel defines the torsional angle $\gamma$. It increases when the methyl group is rotated clockwise. The atom configuration shown corresponds to a small positive $\gamma$ value. Atom positions in the molecule-fixed axis system are shown on the right panel. The three hydrogen atoms of the methyl group are numbered 1, 2, and 3; the hydrogen atom of the hydroxyl group is atom 4 . The $x$ and $z$ axes are those of the RAM axis system. The $x^{\prime}$ and $z^{\prime}$ axes are the principal axes of the polarizability tensor. The configuration shown corresponds to a value of the torsional angle $\gamma$ of $-20^{\circ}$.

nian reviewed by Hougen et al. [23] and based on the work of Hecht and Dennison [24], Kirtman [25], Lees and Baker [26], and Herbst et al. [27]. The RAM Hamiltonian takes the following form:

$$
\begin{aligned}
H_{\mathrm{RAM}} & =F\left(p_{\gamma}-\rho J_{z}\right)^{2}+A J_{z}^{2}+B J_{x}^{2}+C J_{y}^{2} \\
& +D\left\{J_{x}, J_{z}\right\}+V(\gamma),
\end{aligned}
$$

where $p_{\gamma}$ is the momentum conjugated to $\gamma ; J_{x}, J_{y}, J_{z}$ are molecule-fixed components of the rotational angular momentum; $F, \rho, A, B, C, D$ are six kinetic energy parameters; and $V(\gamma)$ is the potential energy function. The RAM Hamiltonian is convenient for numerical calculation because it displays only one rotation-torsion Coriolis coupling term which, as emphasized by Eq. (2), is $2 \rho F p_{\gamma} J_{z}$. The molecule-fixed axis system described above corresponds to the RAM Hamiltonian provided the angle between the molecule-fixed $z$ axis and the axis of internal rotation is suitably chosen. This angle, which should not be confused with the one denoted $\theta_{\text {RAM }}$ introduced by Hougen et al. [23], depends on the molecular geometry. With the geometry proposed by Lees and Baker [26], the present angle is $0.172^{\circ}$ and the values obtained then for the six kinetic energy parameters of the RAM Hamiltonian can be found in Table I. The RAM Hamiltonian does not include distortion effects. These are accounted for adding to this Hamiltonian rotationtorsion terms such as those listed in Table 2 of $\mathrm{Xu}$ et al. [28]. The resulting Hamiltonian may contain more than 100 terms and is the field-free Hamiltonian for the present investigation. It will be denoted $H_{\mathrm{RT}}$. Obtaining actual rotation-torsion energies from $H_{\mathrm{RT}}$ is a two-step process.

In the first step, the so called torsional Hamiltonian [27] $H_{\mathrm{T}}$ is built by retaining in $H_{\mathrm{RT}}$ those terms having diagonal matrix elements between two $|J, k, M\rangle$ symmetric top rotational functions. These functions, defined in Eq. (15.27) of Wigner [29], are eigenfunctions of the 
Table I. Kinetic energy parameters of the RAM Hamiltonian

\begin{tabular}{lrr|lrr}
\hline \hline Parameter $^{\mathrm{a}}$ & Value $^{\mathrm{b}}$ & \multicolumn{1}{c}{ Value $^{\mathrm{c}}$} & Parameter $^{\mathrm{a}}$ & Value $^{\mathrm{b}}$ & Value $^{\mathrm{c}}$ \\
\hline$F$ & 28.1832 & 27.6468 & $B$ & 0.8235 & 0.8236 \\
$\rho$ & 0.8119 & 0.8102 & $C$ & 0.7949 & 0.7925 \\
$A$ & 4.3032 & 4.2537 & $D$ & -0.0025 & -0.0038 \\
\hline \hline
\end{tabular}

a Parameters, defined in Eq. (2), are given in $\mathrm{cm}^{-1}$ except for $\rho$ which is unitless.

b From the geometry of Lees and Baker [26].

c From the analysis of $\mathrm{Xu}$ et al. [28].

total rotational angular momentum $\mathbf{J}^{2}$, of its moleculefixed component $J_{z}$, and of its laboratory-fixed component $J_{Z}$ with eigenvalues $J(J+1), k$, and $M$, respectively. The torsional Hamiltonian $H_{\mathrm{T}}$ depends on the torsional angle $\gamma$ and its matrix can be easily setup using free internal rotation functions $|n\rangle=\exp (i n \gamma) / \sqrt{2 \pi}$ as a basis set. Diagonalization of this matrix yields torsional eigenvalues and eigenfunctions which depend on the rotational quantum numbers $J$ and $k$ as rotational operators in $H_{\mathrm{T}}$ were replaced by their diagonal matrix element prior to the numerical diagonalization. From these eigenfunctions, the symmetry adapted torsional functions in Eqs. (4)-(6) of Coudert and Lopez [21] are deduced. They are characterized by $\Gamma$ their symmetry species in $G_{6}$, the rotational quantum numbers $J$ and $K$, and $v_{t}$ the torsional quantum number. Although the torsional eigenvalues and eigenfunctions do not depend on $M$, as the electric field is not considered as this stage, this quantum number is added for completeness and the torsional functions are written:

$$
\left|\Psi_{J, K, M, v_{t}}^{\Gamma}\right\rangle
$$

where $\Gamma$ is either $A_{1}, A_{2}, E_{a}$, or $E_{b}$. For the two nondegenerate symmetry species, the relations in Section 3.2 of Coudert and Lopez [21] should be used to obtain the symmetry species label from the $A \pm$ label used in Eq. (4) of this reference. For doubly degenerate symmetry species [21], the $a$ and $b$ subscripts identify the component functions obeying the relations $(23)^{*}\left|E_{a}\right\rangle=+\left|E_{a}\right\rangle$ and $(23)^{*}\left|E_{b}\right\rangle=-\left|E_{b}\right\rangle$.

In the second step, rotation-torsion energies and eigenfunctions are computed as in Section 3.2 of Coudert and Lopez [21]. The torsional basis set functions of Eq. (3), with $0 \leq|K| \leq J$ and $0 \leq v_{t} \leq v_{t}^{\mathrm{Max}}$, are used to diagonalize the rotation-torsion Hamiltonian $H_{\mathrm{RT}}$. In this second step, the terms of $H_{\mathrm{RT}}$ which did not appear in the torsional Hamiltonian $H_{\mathrm{T}}$ are taken into account. We are led to evaluate:

$$
\left\langle\Psi_{J, K, M, v_{t}}^{\Gamma}\left|H_{\mathrm{RT}}\right| \Psi_{J, K^{\prime}, M, v_{t}^{\prime}}^{\Gamma}\right\rangle
$$

The nature of torsional basis set functions of Eq. (3) allows us to prediagonalize the rotation-torsion Hamiltonian. The $K=K^{\prime}$ matrix elements in Eq. (4) are nonvanishing only if $v_{t}=v_{t}^{\prime}$. There are no restrictions for the nondiagonal $K \neq K^{\prime}$ matrix elements, but they tend to be small as they involve molecule-fixed components

\begin{tabular}{|c|c|c|c|c|c|c|c|c|}
\hline$J K_{a} K_{c}$ & $\Gamma$ & $v_{t}$ & $E^{\mathrm{b}}$ & $\langle\cos 3 \gamma\rangle^{\mathrm{c}}$ & $\Gamma$ & $v_{t}$ & $E^{\mathrm{b}}$ & $\langle\cos 3 \gamma\rangle^{\mathrm{c}}$ \\
\hline 000 & $A_{1}$ & 0 & $\overline{0.000}$ & 0.537 & $A_{1}$ & 2 & 353.217 & -0.407 \\
\hline 101 & $A_{2}$ & 0 & 1.614 & 0.537 & $A_{2}$ & 2 & 354.825 & -0.407 \\
\hline 111 & $A_{2}$ & 0 & 11.705 & 0.587 & $A_{2}$ & 2 & 476.164 & -0.150 \\
\hline 110 & $A_{1}$ & 0 & 11.733 & 0.587 & $A_{1}$ & 2 & 476.170 & -0.150 \\
\hline 000 & $A_{2}$ & 1 & 294.451 & 0.124 & $A_{2}$ & & 1046.948 & -0.047 \\
\hline 101 & $A_{1}$ & 1 & 296.060 & 0.124 & $A_{1}$ & 3 & 1048.551 & -0.047 \\
\hline 111 & $A_{1}$ & 1 & 227.674 & -0.173 & $A_{1}$ & 3 & 808.868 & -0.069 \\
\hline 110 & $A_{2}$ & 1 & 227.677 & -0.173 & $A_{2}$ & 3 & 808.868 & -0.069 \\
\hline 000 & $E$ & 0 & 9.122 & 0.608 & $E$ & 2 & 510.320 & -0.129 \\
\hline 101 & $E$ & 0 & 10.736 & 0.608 & $E$ & 2 & 511.927 & -0.129 \\
\hline 111 & $E$ & 0 & 16.241 & 0.627 & $E$ & 2 & 556.914 & -0.113 \\
\hline 110 & $E$ & 0 & 5.490 & 0.540 & $E$ & 2 & 371.386 & -0.319 \\
\hline 000 & $E$ & 1 & 208.912 & -0.210 & $E$ & 3 & 751.068 & -0.076 \\
\hline 101 & $E$ & 1 & 210.522 & -0.210 & $E$ & 3 & 752.673 & -0.076 \\
\hline 111 & $E$ & 1 & 204.194 & -0.238 & $E$ & 3 & 705.449 & -0.084 \\
\hline 110 & $E$ & 1 & 288.921 & 0.034 & $E$ & 3 & 990.760 & -0.053 \\
\hline
\end{tabular}

Table II. Calculated rotation-torsion energies ${ }^{\mathrm{a}}$

a Rotation-torsion levels are characterized by the three rotational quantum number $J K_{a} K_{c}$, their symmetry species $\Gamma$ in $G_{6}$, and the torsional quantum number $v_{t}$. Only levels with $J \leq 1$ and $v_{t} \leq 3$ are listed.

b Rotation-torsion energy in $\mathrm{cm}^{-1}$.

${ }^{c}$ Expectation value of $\cos 3 \gamma$.

of the rotational angular momentum. This allows us to truncate the matrix of the rotation-torsion Hamiltonian without accuracy loss. The rotation-torsion energies and wavefunctions thus obtained are written:

$$
E_{J K_{a} K_{c}, M, v_{t}}^{\Gamma} \text { and }\left|\Psi_{J K_{a} K_{c}, M, v_{t}}^{\Gamma}\right\rangle
$$

and characterized by their symmetry species $\Gamma$ in $G_{6}$, the three asymmetric top rotational quantum numbers $J K_{a} K_{c}$, the quantum number $M$, and the torsional quantum number $v_{t}$. In the numerical calculations carried out in this paper, the integer $n_{t}$ in Eq. (2) of Coudert and Lopez [21] was set to 10 and $v_{t}^{\mathrm{Max}}$, defined in the present paper, to 8. A set of spectroscopic parameters is obtained from $\mathrm{Xu}$ et al. [28] and allows us to calculate rotationtorsion energies up to $J=35$ and $v_{t}=3$. The lowest order kinetic energy parameters thus obtained are listed in Table I.

For $J \leq 1$ and $v_{t} \leq 3$, Table II lists calculated rotationtorsion energies and $\langle\cos 3 \gamma\rangle$, the expectation value of $\cos 3 \gamma$, computed using Eq. (A1). This $A_{1}$ symmetry operator, compatible with the 3 -fold symmetry of the methyl group, is a convenient observable for characterizing the torsional function. A large positive value of $\langle\cos 3 \gamma\rangle$ arises when the torsional function is located near $\gamma=0,2 \pi / 3$, and $4 \pi / 3$; a large negative value, when the torsional function is located near $\gamma=\pi / 3$, $\pi$, and $5 \pi / 3$. Table II shows that the largest value of $\langle\cos 3 \gamma\rangle$ is obtained for $v_{t}=0$. For $v_{t} \geq 1$, the expectation value depends on the rotational and torsional quantum numbers and on the symmetry species. Within a given torsional manifold, the fast variation with the rotational quantum number stems from strong rotationtorsion Coriolis coupling in methanol, studied and suc- 


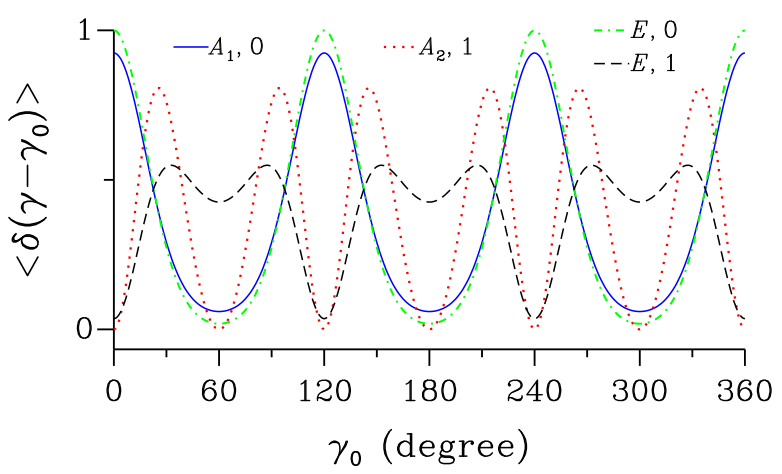

Figure 4. Variations of $\left\langle\delta\left(\gamma-\gamma_{0}\right)\right\rangle$ the expectation value of $\delta\left(\gamma-\gamma_{0}\right)$ as a function of $\gamma_{0}$ in degrees for 4 rotationtorsion levels with $J=0$. Solid, dotted, dotted-dashed, and dashed lines correspond to levels with $\left(\Gamma, v_{t}\right)=\left(A_{1}, 0\right)$, $\left(A_{2}, 1\right),(E, 0)$, and $(E, 1)$, respectively.

cessfully modeled in the early spectroscopic investigations of this molecule [30]. Between torsional manifolds, the variation can be understood with the help of Fig. 4 showing the torsional dependence of the squared rotation-torsion wavefunction calculated as the expectation values of the operator $\delta\left(\gamma-\gamma_{0}\right)$. The expectation value of this operator, involving the Dirac delta function, was evaluated using Eqs. (A2) and (A3). For $v_{t}=0$, Fig. 4 is consistent with torsional functions centered near $\gamma=0,2 \pi / 3$, and $4 \pi / 3$ leading to a large value of $\langle\cos 3 \gamma\rangle$. For $v_{t}=1$, the $E$-symmetry torsional functions are centered near $\gamma=\pi / 3, \pi$, and $5 \pi / 3$, resulting in a negative value of $\langle\cos 3 \gamma\rangle$, while the $A_{2}$-symmetry torsional function vanishes for $\gamma=0, \pi / 3,2 \pi / 3, \pi, 4 \pi / 3$, and $5 \pi / 3$, leading to a small value of $\langle\cos 3 \gamma\rangle$.

\section{B. Stark coupling Hamiltonian}

When the methanol molecule is subject to a nonresonant laser field, the Stark coupling Hamiltonian $H_{\mathrm{S}}(t)$ describing the interaction with the electric field takes the form:

$$
H_{\mathrm{S}}(t)=-\frac{1}{2} \mathcal{E}(t) \cdot \boldsymbol{\alpha}(\gamma) \cdot \mathcal{E}(t)
$$

where $\mathcal{E}(t)$ is the laser electric field and $\boldsymbol{\alpha}(\gamma)$ is the $3 \times 3$ $\gamma$-dependent dynamical polarizability tensor, including contribution of the excited electronic states. Taking the laser field polarized in the $Z$ direction, the electric field is then $\mathcal{E}(t)=\mathcal{E}(t) \cos \omega t \mathbf{i}_{Z}$, where $\mathcal{E}(t)$ is the electric field envelope, $\omega$ is the optical frequency, and $\mathbf{i}_{Z}$ is the unit vector along the space-fixed $Z$ axis. When the optical frequency is far detuned from all vibronic transitions, as in the present investigation, the dynamical polarizability [31-33] is well approximated by the static one. Additionally, in the case of a slowly varying electric field envelope [31-33], the Stark coupling Hamiltonian can be time averaged leading to:

$$
H_{\mathrm{S}}(t)=-\frac{1}{4} \mathcal{E}(t)^{2} \sum_{i, j} \Phi_{Z i} \Phi_{Z j} \alpha_{i j}(\gamma)
$$

where $i, j$ span the molecule-fixed axis system; $\Phi_{Z i}$ is a direction cosine; and $\alpha_{i j}(\gamma)$ are molecule-fixed components of the static polarizability tensor. Symmetry considerations [23] show that these components should be expanded in terms of the torsional angle $\gamma$ as:

$$
\alpha_{i j}(\gamma)=\sum_{k=0}^{p} \alpha_{i j}^{k} \cos 3 k \gamma
$$

when $i j=x x, y y, z z$, and $x z$; and

$$
\alpha_{i j}(\gamma)=\sum_{k=1}^{p} \beta_{i j}^{k} \sin 3 k \gamma,
$$

when $i j=x y$ and $y z$. In these two equations, $\alpha_{i j}^{k}$ and $\beta_{i j}^{k}$ are expansion coefficients. Truncating the expansions to $p=2$, numerical values for these coefficients were retrieved fitting the polarizability tensor components calculated, with the help of ab initio calculations, by Davis and Dennison [34] for several values of of $\gamma$. The results of these authors obtained with the Spackman basis set were used and a transformation corresponding to a $0.172^{\circ}$ rotation about the molecule-fixed $y$ axis was performed, as in Section III A, because these authors used the molecule-fixed axis system of Lees and Baker [26] instead of the RAM axis system. Numerical values for $\alpha_{i j}^{k}$ and $\beta_{i j}^{k}$ are listed in Table III. This table emphasizes that the parameters with $k \geq 1$, describing the torsional dependence, are very small. They will be neglected in the remainder of the paper and this leads to a constant polarizability tensor with nonvanishing $x x, y y, z z$, and $x z$ molecule-fixed components equal to $\alpha_{x x}^{0}, \alpha_{y y}^{0}, \alpha_{z z}^{0}$, and $\alpha_{x z}^{0}$, respectively. The principal axes of the polarizability tensor, denoted $x^{\prime} y^{\prime} z^{\prime}$, are such that the $y$ and $y^{\prime}$ axes are parallel; the angle between the $x$ and $x^{\prime}$ axes being $18.17^{\circ}$, as emphasized by Fig. 3. In the $x^{\prime} y^{\prime} z^{\prime}$ axis system, the diagonal components of the polarizability tensor, $\alpha_{x^{\prime}}, \alpha_{y^{\prime}}, \alpha_{z^{\prime}}$, are equal to $2.7010,2.5867$, and $3.1662 \AA^{3}$, respectively. In the high intensity limit, the $z^{\prime}$ axis, associated with the largest component, is aligned along the space-fixed $Z$ axis leading to $\Phi_{Z x}^{2}=0.09723$, $\Phi_{Z y}^{2}=0, \Phi_{Z z}^{2}=0.90277$, and $\Phi_{Z x} \Phi_{Z z}=0.29627$.

Using as a basis set the field-free rotation-torsion wavefunctions in Eq. (5), the matrix of the Stark coupling Hamiltonian is setup leading to the matrix elements:

$$
\left\langle\Psi_{J K_{a} K_{c}, M, v_{t}}^{\Gamma}\left|H_{\mathrm{S}}(t)\right| \Psi_{J^{\prime} K_{a}^{\prime} K_{c}^{\prime}, M^{\prime}, v_{t}^{\prime}}^{\Gamma^{\prime}}\right\rangle .
$$

Owing to the fact that $H_{\mathrm{S}}(t)$ belongs to the completely symmetrical $A_{1}$ symmetry species of $G_{6}$, this matrix element is nonvanishing if $\Gamma=\Gamma^{\prime}$. In agreement with Section 3.2 of Coudert and Lopez [21], we are then led to 
Table III. Polarizability tensor expansion coefficients ${ }^{\mathrm{a}}$

\begin{tabular}{r|rrrrrr}
\hline \hline$k$ & $\alpha_{x x}^{k}$ & \multicolumn{1}{c}{$\alpha_{y y}^{k}$} & \multicolumn{1}{c}{$\alpha_{z z}^{k}$} & \multicolumn{1}{c}{$\alpha_{x z}^{k}$} & $\beta_{x y}^{k}$ & \multicolumn{1}{c}{$\beta_{y z}^{k}$} \\
\hline 0 & 2.7454 & 2.5867 & 3.1218 & 0.1367 & & \\
1 & 0.0064 & 0.0061 & -0.0004 & -0.0064 & -0.0005 & -0.0008 \\
2 & 0.0020 & -0.0007 & 0.0026 & 0.0027 & -0.0079 & 0.0000 \\
\hline \hline
\end{tabular}

a Numerical values in $\AA^{3}$ for the expansion coefficients in

Eqs. (8) and (9) were obtained from Davis and Dennison [34]

evaluate the matrix elements of $H_{\mathrm{S}}(t)$ between two basis set torsional functions of Eq. (3):

$$
\left\langle\Psi_{J, K, M, v_{t}}^{\Gamma}\left|H_{\mathrm{S}}(t)\right| \Psi_{J^{\prime}, K^{\prime}, M^{\prime}, v_{t}^{\prime}}^{\Gamma}\right\rangle .
$$

Equations (1)-(3) of Coudert and Lopez [21], and Eq. (7) of the present paper show that Eq. (11) leads to matrix elements of direction cosines between two symmetric top rotational functions [29] and to torsional matrix elements of polarizability tensor components. They take, respectively, the following form:

$$
\left\langle J, k, M\left|\Phi_{Z i} \Phi_{Z j}\right| J^{\prime}, k^{\prime}, M^{\prime}\right\rangle
$$

and

$$
\left\langle n\left|\alpha_{i j}(\gamma)\right| n^{\prime}\right\rangle
$$

where $|n\rangle$ is a free internal rotation function as defined in Section III A. The matrix element in Eq. (12) can be calculated using tensorial operator algebra [35] as in Coudert [36]. Because in this equation both direction cosines correspond to the space-fixed $Z$ axis, the restriction $M=M^{\prime}$ holds. The matrix element in Eq. (13) is nonzero if $i j=x x, y y, z z$, or $x z$ and reduces then to $\alpha_{i j}^{0} \delta_{n, n^{\prime}}$.

Equation (10) means that the matrix of the Stark Hamiltonian can be split into four blocks corresponding to the symmetry species of $G_{6}$. Equation (12) shows that each block can be further split into several subblocks characterized by $M$.

\section{Dissipation}

In order to describe the dissipation process in the experimental alignment signal presented in Section II, the density matrix formalism is adopted [20, 37, 38]. The density operator $\rho(t)$ is governed by the quantum Liouville-von Neumann equation:

$$
\frac{\partial \rho(t)}{\partial t}=-\frac{i}{\hbar}\left[H_{\mathrm{RT}}+H_{\mathrm{S}}(t), \rho(t)\right]+\left(\frac{\partial \rho(t)}{\partial t}\right)_{\mathrm{diss}}
$$

where the last term is the dissipative operator. The experimental signal described in Section II is consistent with a dissipation process which barely alters the permanent alignment but leads to a fast decrease of the transient alignment. An analogous dissipation process has been experimentally evidenced in the linear $\mathrm{CO}_{2}$ molecule and was theoretically modeled using an $M / J$ conserving model [20]. In the present work, we only consider in Eq. (14) the dissipation of the transient alignment that is approximated by:

$$
\left(\frac{\partial \rho(t)_{i, j}}{\partial t}\right)_{\mathrm{diss}}= \begin{cases}-\rho(t)_{i, j} / \tau, & i \neq j \\ 0, & i=j\end{cases}
$$

where $i, j$ are two field-free rotation-torsion levels of Eq. (5); and $\tau$ is a decay time constant assumed to be the same for all pairs of levels. Although this choice cannot be theoretically substantiated, it is the simplest one and leads to straightforward numerical calculations.

\section{NUMERICAL RESULTS}

The effects of a laser field with a constant intensity are investigated first computing the intensity variation of various thermal averages. The time evolutions of the same averages, after submitting the molecule to the laser pulse, are calculated afterwards. The first calculation does not correspond to an actual experiment and was carried out to understand the effects of the laser. The following calculations correspond to the experiment described in Section II and allows us to compare experimental and theoretical alignment signals.

In the calculations below, the field-free rotation-torsion wavefunctions of Eq. (5) are used as basis set functions. The maximum values of the rotational quantum number $J$ and of the torsional quantum number $v_{t}$ are $J_{\text {Max }}=28$ and $v_{t \text { Max }}=3$, respectively. When evaluating thermal averages, the statistical weights were taken equal to 4 for all three symmetry species of $G_{6}$ [39].

To understand the results of the time evolution calculations, a comparison with those derived for a fictitious rigid molecule is made. This molecule is chosen so that its rotational energy levels are the closest to those of methanol. Its rotational Hamiltonian $H_{\mathrm{R}}$, deduced from Eq. (2), reads:

$$
H_{\mathrm{R}}=A J_{z}^{2}+B J_{x}^{2}+C J_{y}^{2}+D\left\{J_{x}, J_{z}\right\},
$$

where the spectroscopic parameters $A, B, C$, and $D$ are set to the values retrieved from $\mathrm{Xu}$ et al. [28], listed in Table I. The polarizability tensor of the rigid molecule is the one chosen for methanol in Section IIIB.

\section{A. Static alignment}

Assuming a time-independent laser field envelope $\mathcal{E}(t)=\mathcal{E}_{0}$, the full rotation-torsion Hamiltonian plus the time-independent Stark coupling Hamiltonian $H_{\mathrm{RT}}+H_{\mathrm{S}}$ was diagonalized taking the field-free rotation-torsion wavefunctions of Eq. (5) as basis set functions. This leads to diagonal matrix elements for $H_{\mathrm{RT}}$ while those 

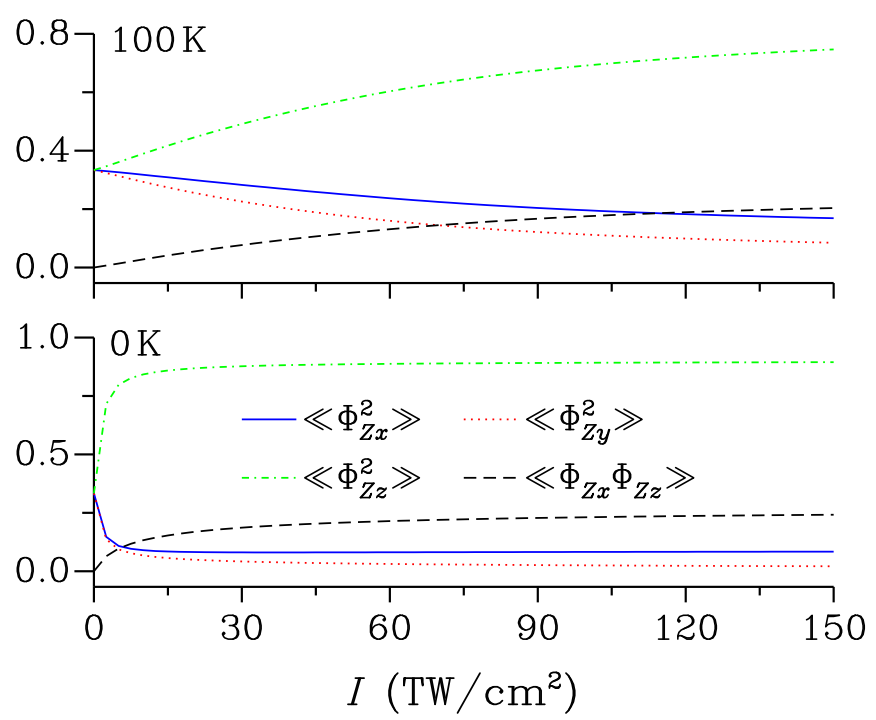

Figure 5. The thermal averages $\left\langle\left\langle\Phi_{Z x}^{2}\right\rangle\right\rangle,\left\langle\left\langle\Phi_{Z y}^{2}\right\rangle\right\rangle,\left\langle\left\langle\Phi_{Z z}^{2}\right\rangle\right\rangle$, and $\left\langle\left\langle\Phi_{Z x} \Phi_{Z z}\right\rangle\right\rangle$ are plotted in solid, dotted, dotted-dashed, and dashed lines, respectively, as functions of the laser beam intensity in $\mathrm{TW} / \mathrm{cm}^{2}$. The temperatures are 0 and $100 \mathrm{~K}$ for the lower and upper panels, respectively. The $y$ axis scales are not the same for the two panels.

of $H_{\mathrm{S}}$ are evaluated using Section IIIB. Stark energies and eigenvalues were retrieved for each symmetry species of $G_{6}$ and for each value of the quantum number $M$. The maximum value of the laser beam intensity was $150 \mathrm{TW} / \mathrm{cm}^{2}$. Thermal averages were computed assuming a thermal ensemble of molecules described by a Boltzmann distribution before the laser field is turned on and an adiabatic transfer of populations [40] when the intensity of the laser field increases. Methanol being an asymmetric top molecule, the three thermal averages $\left\langle\left\langle\Phi_{Z x}^{2}\right\rangle\right\rangle,\left\langle\left\langle\Phi_{Z y}^{2}\right\rangle\right\rangle$, and $\left\langle\left\langle\Phi_{Z z}^{2}\right\rangle\right\rangle$ were computed. As the rotation-torsion Hamiltonian contains terms in $\left\{J_{x}, J_{z}\right\}$, as emphasized by Eq. (2), the thermal average $\left\langle\left\langle\Phi_{Z x} \Phi_{Z z}\right\rangle\right\rangle$ is nonvanishing and needs also to be computed. Figure 5 depicts the intensity variations of the $\left\langle\left\langle\Phi_{Z x}^{2}\right\rangle\right\rangle,\left\langle\left\langle\Phi_{Z y}^{2}\right\rangle\right\rangle,\left\langle\left\langle\Phi_{Z z}^{2}\right\rangle\right\rangle$, and $\left\langle\left\langle\Phi_{Z x} \Phi_{Z z}\right\rangle\right\rangle$ for two temperatures, zero and $100 \mathrm{~K}$. For the zero temperature, Fig. 5 is consistent with the molecule-fixed $z^{\prime}$ axis becoming aligned along the space-fixed $Z$ axis for an intensity larger than $50 \mathrm{TW} / \mathrm{cm}^{2}$. Beyond this intensity value, the thermal averages $\left\langle\left\langle\Phi_{Z x}^{2}\right\rangle\right\rangle,\left\langle\left\langle\Phi_{Z y}^{2}\right\rangle\right\rangle,\left\langle\left\langle\Phi_{Z z}^{2}\right\rangle\right\rangle$, and $\left\langle\left\langle\Phi_{Z x} \Phi_{Z z}\right\rangle\right\rangle$ are equal to $0.0849,0.0213,0.8938$, and 0.2437 , respectively, and are close to the values retrieved in Section III B in the high intensity limit. For the $100 \mathrm{~K}$ temperature, Fig. 5 shows that, as expected [41], the alignment is reduced compared to $0 \mathrm{~K}$.

The intensity variations of the thermal average $\langle\langle\cos 3 \gamma\rangle\rangle$ can be seen in Fig. 6 for temperatures of zero, 100 , and $295 \mathrm{~K}$. For the lowest temperature, a small increase of 0.035 can be seen while larger decreases of 0.110 and 0.066 arise for 100 and $295 \mathrm{~K}$, respectively. Although theses variations cannot be easily understood, Table II
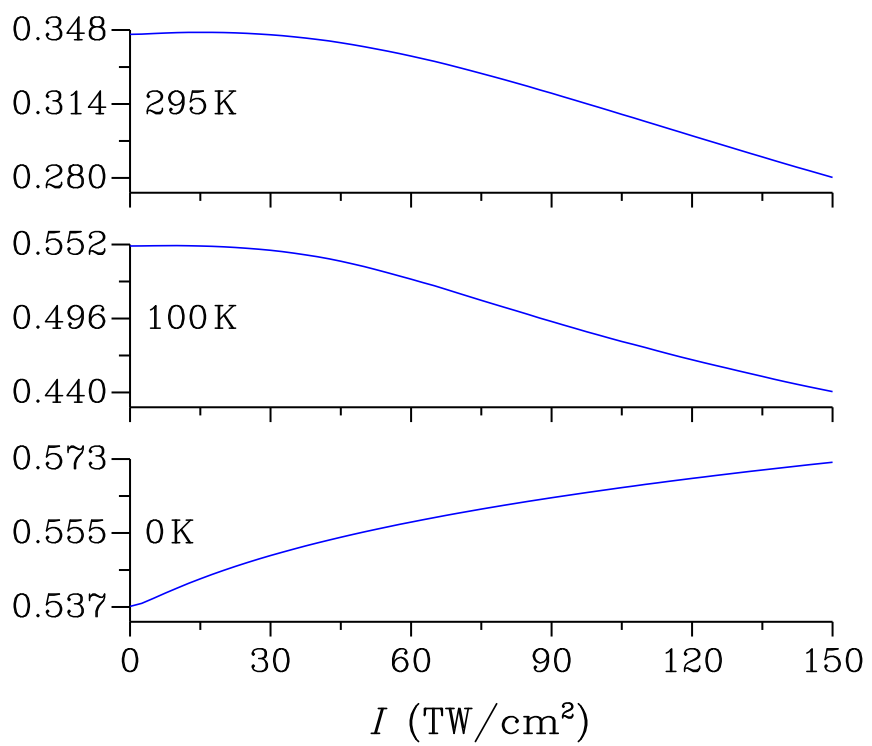

Figure 6. The thermal average $\langle\langle\cos 3 \gamma\rangle\rangle$ is plotted as a function of the laser beam intensity in $\mathrm{TW} / \mathrm{cm}^{2}$. The temperatures are 0,100 , and $295 \mathrm{~K}$ for the lower, middle, and upper panels, respectively. The $y$ axis scales are not the same for the three panels.

shows that the low-lying levels populated at low temperature are characterized by a value of $\langle\cos 3 \gamma\rangle$ close to 0.5 . For a higher temperature, higher lying rotationtorsion levels with a negative value of $\langle\cos 3 \gamma\rangle$ become populated and this might explain the decrease and the larger variation of the thermal average.

\section{B. Laser pulse effects}

The molecule is subject to the Gaussian laser pulse described in Section II. Dissipation effects are ignored and the wavefunctions are expanded with the help of the field-free rotation-torsion wavefunctions of Eq. (5). In this case too, the nature of the Stark coupling Hamiltonian $H_{\mathrm{S}}(t)$ allows us to simplify the calculation as each symmetry species and each $M$-value can be propagated separately. During the laser pulse, a time grid [42] and the Chebychef scheme $[43,44]$ are used. The pulse is assumed to take place at $t_{0}=0$, and wavefunctions are propagated from $t=-5 \mathrm{ps}$ to $t=100 \mathrm{ps}$. The intensity of the laser pulse was assumed vanishingly small outside the $\left[-t_{p},+t_{p}\right]$ time interval. Taking $t_{p}$ equal to $0.25 \mathrm{ps}$ ensures that outside this interval the intensity of the laser pulse remains smaller than $200 \mathrm{MW} / \mathrm{cm}^{2}$. From $t=$ -5 ps to $t=-t_{p}$, a field-free time evolution is used; from $t=-t_{p}$ to $t=+t_{p}$ ps, the time-dependent Schrödinger equation is solved using the Chebychef scheme; and from $t=+t_{p}$ to $t=100 \mathrm{ps}$, a field-free time evolution is used again. The time evolution of the thermal average $\left\langle\left\langle\Phi_{Z z}^{2}\right\rangle\right\rangle$, shown in Fig. 7, was thus computed for an ensemble of molecules described by a Boltzmann distribution charac- 


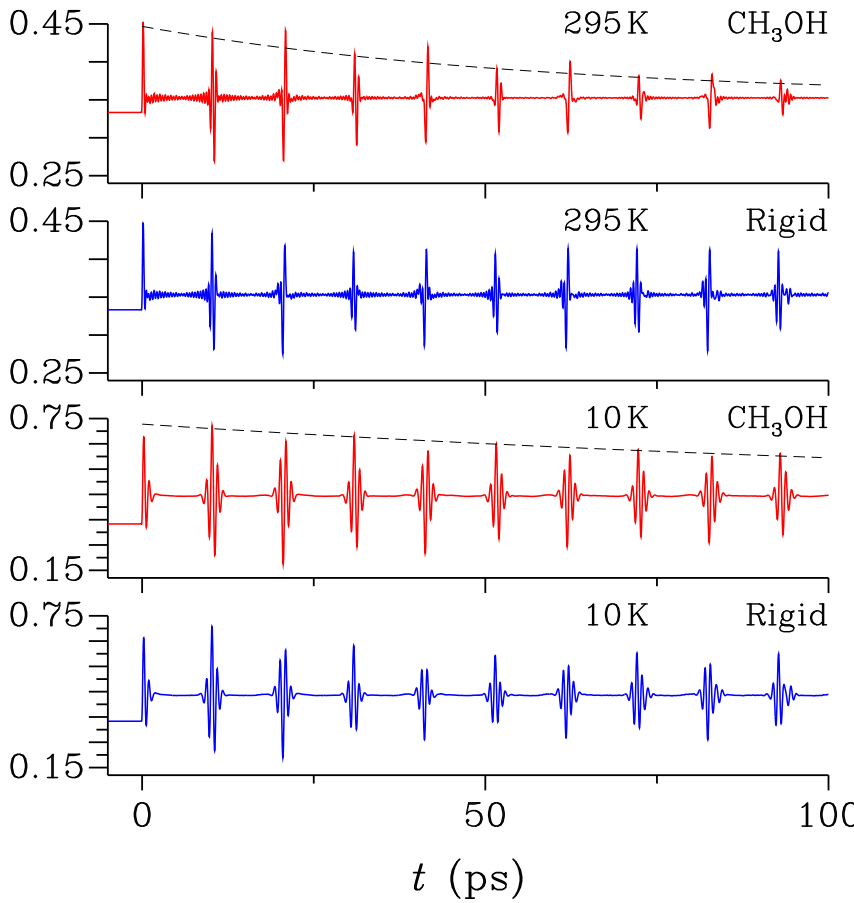

Figure 7. Variations of the thermal average $\left\langle\left\langle\Phi_{Z z}^{2}\right\rangle\right\rangle$ as a function of the time $t$ in ps. At $t=0$, the molecule is subject to a $140 \mathrm{TW} / \mathrm{cm}^{2}$ laser pulse with $0.1 \mathrm{ps}$ duration. The temperatures are 10 and $295 \mathrm{~K}$ for the two lower and two upper panels, respectively. The $1^{\text {st }}$ and $3^{\text {rd }}\left(2^{\text {nd }}\right.$ and $\left.4^{\text {th }}\right)$ panels from the bottom correspond to the equivalent rigid (methanol) molecule. For the methanol molecule, the dashed lines show exponential decays with decay times of 157.9 and $57.4 \mathrm{ps}$ for the 10 and $295 \mathrm{~K}$ temperatures, respectively. The $y$ axis scales are different for the four panels.

terized by a temperature of either 10 or $295 \mathrm{~K}$ before the pulse. Figure 7 also displays the time evolutions for the rigid molecule equivalent to methanol.

From -5 to $50 \mathrm{ps}$, the thermal averages of the methanol and equivalent rigid molecule exhibit, for both temperatures, very similar time variations. A permanent alignment appears after the pulse along with a transient alignment characterized by revivals with a periodicity equal to $1 /[2(B+C)]=10.32 \mathrm{ps}$ and a complicated form characteristic of asymmetric-top molecules [45]. From 50 to $100 \mathrm{ps}$, the peak alignment amplitude decreases for methanol while it barely changes for the equivalent rigid molecule. This can be understood remembering that in methanol an accurate picture of the rotation-torsion energies $[46,47]$ can be obtained using a rotational constant $B\left(\Gamma, K, v_{t}\right)$ for each torsional level. Since, as emphasized by Eq. (16) of Zhang et al. [19], the periodicity of the transient alignment depends on the value of the rotational constant $B$, the time evolution of the alignment signal will contain contributions with slightly different periodicity due to the dependence of the $B$ rotational constant on the torsional level. The resulting gradual dephasing leads to an exponential decrease of the peak alignment [19]. The total number of torsional levels con-
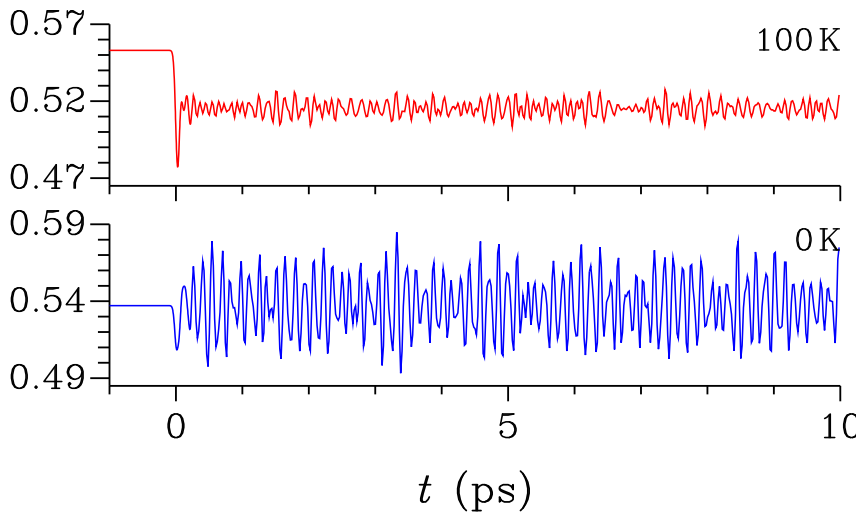

Figure 8. Variations of the thermal averages $\langle\langle\cos 3 \gamma\rangle\rangle$ as a function of the time $t$ in ps. At $t=0$, the molecule is subject to a $140 \mathrm{TW} / \mathrm{cm}^{2}$ laser pulse with $0.1 \mathrm{ps}$ duration. The temperatures are 0 and $100 \mathrm{~K}$ for the lower and upper panels, respectively. The $y$ axis scale are not the same for the two panels.

sidered amounts to:

$$
2\left(v_{t \operatorname{Max}}+1\right)\left(2 J_{\operatorname{Max}}+1\right)
$$

for $M=0$. With the values of $v_{t \text { Max }}$ and $J_{\text {Max }}$ used, the number of torsional states is 456 . This number does not take into account level population. For low temperatures, only a fraction of torsional levels are populated and dephasing effects should be smaller. This is confirmed by the present calculation leading to decay time constants of 157.9 and 57.4 ps for 10 and $295 \mathrm{~K}$, respectively. The corresponding decay curves are plotted in Fig. 7.

Figure 8 depicts the time evolution of the thermal average $\langle\langle\cos 3 \gamma\rangle\rangle$ computed for an ensemble of molecules described by temperatures of 0 and $100 \mathrm{~K}$ before the pulse. For the lower temperature semi periodic variations with a fundamental periodicity of $0.137 \mathrm{ps}$ can be seen. This periodicity corresponds to an energy of $243 \mathrm{~cm}^{-1}$ close to the energy difference between the ground and first excited torsional states. For the higher temperature, semi periodic variations also arise, but their amplitude is much smaller. A permanent torsional alignment can clearly be seen as the thermal average is 0.552 before the pulse and 0.515 after. An analogous permanent alignment was also evidenced for the torsional angle of a biphenyl molecule [48] subject to a strong laser pulse.

\section{Experimental alignment analysis}

The molecule is subject to the Gaussian laser pulse described in Section II and used in the previous section. Since dissipation effects are now taken into account, the density matrix formalism is used. During the laser pulse, from $t=-t_{p}$ to $t=+t_{p}$, the time dependent Liouvillevon Neumann equation of Eq. (14) is solved and the density matrix is propagated using a time grid and a version 
of the Chebychef scheme appropriate for the density matrix [49]. From $t=-5$ to $t=-t_{p}$ ps, before the pulse, and from $t=+t_{p}$ to $t=57 \mathrm{ps}$, after the pulse, the electric field is vanishingly small and in Eq. (14) the Stark coupling Hamiltonian $H_{\mathrm{S}}(t)$ can be neglected. Remembering the assumptions made in Section III C, we find that for any two times $t$ and $t^{\prime}$, both before or after the pulse, the density matrix is given exactly by:

$$
\rho(t)_{i, j}= \begin{cases}\rho\left(t^{\prime}\right)_{i, j} e^{\left[i\left(E_{i}-E_{j}\right) / \hbar-1 / \tau\right]\left(t-t^{\prime}\right)}, & i \neq j \\ \rho\left(t^{\prime}\right)_{i, j}, & i=j\end{cases}
$$

where $i$ and $j$ are field-free rotation-torsion levels of Eq. (5); and $E_{i}$ and $E_{j}$ their energy. At $t=-5 \mathrm{ps}$, the density matrix is that of a thermal equilibrium; its matrix elements are given by $\rho(t)_{i, j}=\delta_{i, j} e^{-E_{i} / k T} / Z$, where $T$ is the temperature, $k$ the Boltzmann's constant, and $Z$ the partition function.

As already mentioned in Section II, the experimental signal is proportional to $\Delta n$, the difference of refractive indices which is defined in Eq. (9) of Rouzée et al. [45]. It depends on the direction cosines and on the polarizability tensor:

$$
\begin{aligned}
\Delta n & =\frac{3 N}{4 n \epsilon_{0}}\left[\alpha_{x x}^{0}\left(\Phi_{Z x}^{2}-\frac{1}{3}\right)+\alpha_{y y}^{0}\left(\Phi_{Z y}^{2}-\frac{1}{3}\right)\right. \\
& \left.+\alpha_{z z}^{0}\left(\Phi_{Z z}^{2}-\frac{1}{3}\right)+2 \alpha_{x z}^{0} \Phi_{Z x} \Phi_{Z z}\right]
\end{aligned}
$$

where $n$ is the average value of the refractive index at the probe frequency, $N$ the number density, and $\epsilon_{0}$ the dielectric constant of vacuum. At thermal equilibrium, without the laser field, $\Delta n$ is zero. It becomes nonzero after the laser pulse.

Assuming a temperature of $295 \mathrm{~K}$, the density matrix was propagated and the time evolution of the difference of refractive indices was computed accounting for the torsion with the model in Section III and using the equivalent rigid molecule model in Section IV. In agreement with Section II, both calculated signals were convoluted with a Gaussian function with a HWHM of 0.1 ps. The best match between experimental and calculated alignment signals was achieved adjusting the decay time constant $\tau$, introduced in Section III C, and the non-diagonal component $\alpha_{x z}^{0}$ of the polarizability tensor. The adjustment was carried out manually cycling through the values of either parameter with a step size of 0.1 ps for $\tau$ and $0.001 \AA^{3}$ for $\alpha_{x z}^{0}$. The experimental alignment signal was first multiplied by a factor such that both experimental and theoretical signals are identical outside the revivals, that is, making sure the experimental and theoretical permanent alignment are the same. In agreement with Fig. 2, this fitting was carried out from 3 to 8 ps, from 12 to $18 \mathrm{ps}$, etc. Changing then $\alpha_{x z}^{0}$ allowed us to alter the peak amplitude of the transient alignment. $\alpha_{x z}^{0}$ was thus determined matching experimental and theoretical signals for the first revival at $t=10.4$ ps. $\tau$ was subsequently obtained exploiting the experimental signal at the second, third, and fourth revivals, more precisely, $\tau$ was changed so as to improve the agreement near $t=21$, 31.5 and $42 \mathrm{ps}$. Changing $\tau$ alters the most the calculated signal at the fourth revival and to a lesser extent that near the first revival. The value of $\alpha_{x z}^{0}$ was therefore slightly refined again. The final fit was obtained after a last refinement of $\tau$. Figure 9 shows a comparison between the experimental and the two calculated signals. The largest discrepancies can be seen at the revivals. One issue being that the intensity of all five revivals could not be simultaneously reproduced, especially in the case of the equivalent rigid molecule model. A conservative estimate of the parameter uncertainty is $10 \%$. With the model taking the torsion into account, we obtained $\tau=83.3 \mathrm{ps}$ and $\alpha_{x z}^{0}=0.369 \AA^{3}$. The non-zero value retrieved for $\tau$ implies that the observed decay results from both the torsional motion and the collisional dissipation. The fitted non-diagonal component $\alpha_{x z}^{0}$ is 3 times larger than that deduced from Davis and Dennison [34]. With the equivalent rigid molecule model, a shorter decay time constant $\tau$ of $35 \mathrm{ps}$ and a non-diagonal component $\alpha_{x z}^{0}$ equal to $0.232 \AA^{3}$ were retrieved. With this model the latter is only 1.7 times larger than that deduced from Davis and Dennison [34]. Figure 9 emphasizes that the best agreement is achieved when the torsion is accounted for. In this case, both the shapes of the alignment transients and their decay are well reproduced. In contrast, the simplified equivalent rigid molecule model is less accurate, and furthermore small features at 4.5 and 14 ps arise which have no counterpart in the experimental signal.

\section{DISCUSSION}

The behavior of a non-rigid methanol molecule subject to a strong laser pulse was studied experimentally and theoretically. An experimental alignment signal was recorded and analyzed with the help of a theoretical approach accounting for the overall rotation, the large amplitude internal rotation of the methyl group, and the Stark coupling.

The theoretical treatment is based on an effective four dimensional rotation-torsion Hamiltonian in which the overall rotation and the large amplitude torsional motion of the methyl group are treated simultaneously [2327]. This treatment, presented in Section III A, allows us to reproduce accurately the field-free rotation-torsion energy levels [28]. The coupling of the molecule with the nonresonant laser field is described by a second order Stark coupling involving a polarizability tensor depending, in principle, on the large amplitude torsional angle $\gamma$. Ab initio calculations [34] revealed that this dependence, very weak, can be ignored. In Section IV A, a theoretical calculation of the effects of a c.w. laser field is carried out. It was found that rotational and torsional alignments take place. The latter, described by the thermal average $\langle\langle\cos 3 \gamma\rangle\rangle$, increases or decreases when the 

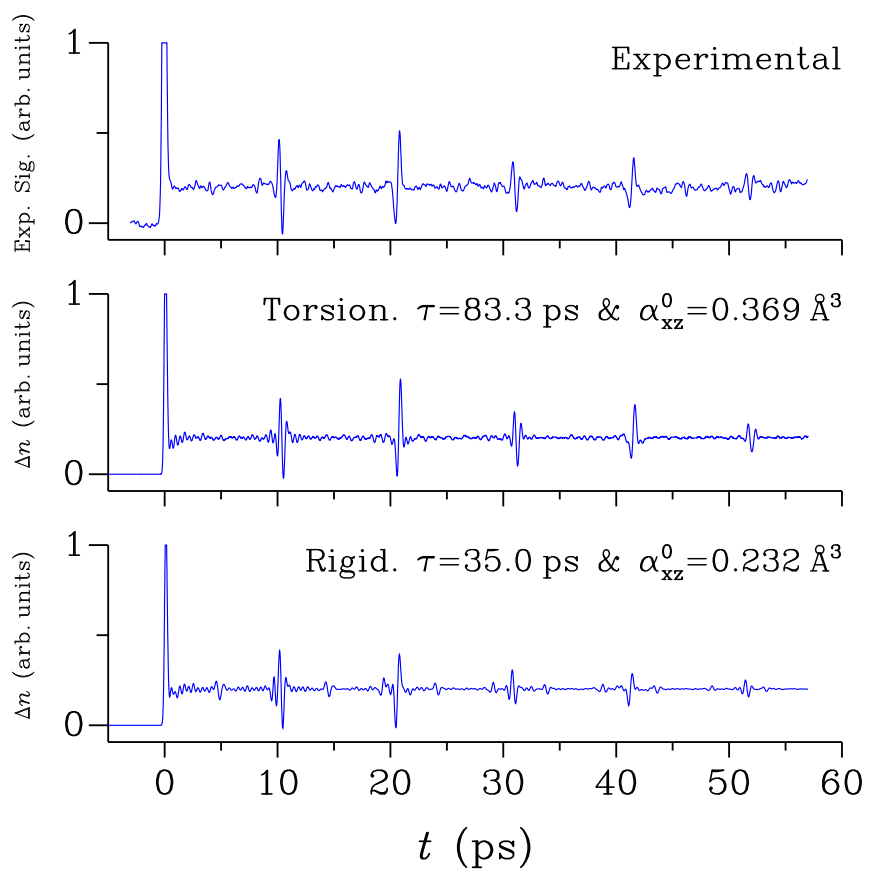

Figure 9. The experimental alignment signal, upper panel, is compared to the difference of refractive indices $\Delta n$ calculated taking the torsion into account, middle panel, and using the equivalent rigid molecule model, lower panel. In both calculations, the decay time constant $\tau$ and the non-diagonal component $\alpha_{x z}^{0}$ of the polarizability tensor were adjusted.

strength of the laser field increases, depending on the temperature. This result, unexpected, since the Stark coupling Hamiltonian does not depend explicitly on the large amplitude torsional coordinate, stems from the fact that the torsional energy levels of a methyl group attached to a freely rotating hydroxyl group differ from those of a methyl group attached to an hydroxyl group aligned along the electric field. Unlike in biphenyl-like molecules [4-14], the electric field does not alter the torsional potential energy function, but changes a torsional kinetic energy term. In Section IV B, the effects of the strong short pulse are computed for several temperatures. The theoretical approach shows that a permanent and a transient rotational alignment arise. The latter is similar to that of a rigid molecule for the first five revivals. For the next revivals, an exponential decay of the maximum amplitude of the transient alignment takes place and is due to the high density of states arising from the large amplitude torsional motion. The time decay constants deduced from the calculation were found to be 157.9 and 57.4 ps for temperatures of 10 and $295 \mathrm{~K}$, respectively. Permanent and transient torsional alignment were also calculated. The former (latter) increases (decreases) as the temperature rises. The effects of the torsion, calculated in this work for the transient alignment, are also important for other dynamical aspects of methanol. The torsion was shown to be a contributing factor for the short timescales of the intramolecular vibrational energy transfer [50] observed for high-lying vibrational states of methanol.

The main results of this work are presented in Section IV C where experimental and theoretical alignment signals are compared. Two theoretical signals were computed. For the first one, methanol was treated as a rigid molecule using the equivalent rigid molecule model of Section IV. For the second one, methanol was treated as a non-rigid molecule undergoing internal rotation and the approach developed in Section III was utilized. With both calculations, a satisfactory agreement between experiment and theory was achieved increasing the value of the nondiagonal component of the polarizability tensor and adding to the theoretical model dissipation effects for the transient alignment only. The value obtained for the decay constants was 35 ps when the molecule is assumed rigid and $83.3 \mathrm{ps}$ when the torsion is taken into account. The longer decay obtained in this case means that the experimental decay contains contributions from both molecular collisions and the torsion. The decay constant retrieved in Section IV B for that effect being 57.4 ps, the decay constant resulting from both effects is $34 \mathrm{ps}$ and agrees well with that obtained with the rigid molecule model. Figure 9, where a comparison between the experimental alignment signal and the theoretical ones is shown, emphasizes that despite the parameter adjustment a better agreement arises with the model where the internal rotation is taken into account.

Methanol is a benchmark molecule for studying the hindered rotation of a methyl group. This torsion is well understood in high-resolution spectroscopy [26-28, 30] and the present investigation is consistent with its effect being also noticeable in birefringence experiments. These effects could not be unambiguously evidenced because the recorded experimental signal turns out to be very close to that of a rigid slightly asymmetric prolate top. The internal rotation effects could be conclusively evidenced if an alignment signal was recorded at a very low pressure to reduce dissipation effects due to molecular collisions. The experimental signal should also be recorded for at least 100 ps so that the decay due solely to internal rotation, described in Section IV B, is measured. Furthermore, an effective control of the methyl group torsion could be confirmed designing an experiment allowing us to measure the thermal average $\langle\langle\cos 3 \gamma\rangle\rangle$. With the fitted value of the non-diagonal component of the polarizability tensor, a substantial change of the permanent torsional alignment is calculated at $295 \mathrm{~K}$ as it decreases from 0.346 before the pulse to 0.261 after.

\section{ACKNOWLEDGMENTS}

This work was supported by the Conseil Régional de Bourgogne (PARI program), the European Union (FEDER program), and the CNRS. The authors gratefully acknowledge S. Pernot and B. Sinardet for their help in the balanced detection setup. The authors are 
very indebted to Vadim Ilyushin for providing them with the list of calculated energy levels of methanol.

\section{Appendix A: Torsional matrix elements and expectation values}

$\left\langle n|O| n^{\prime}\right\rangle$ the matrix elements of the torsional operator $O$ between two free internal rotation functions of Section III A are listed for $O=p_{\gamma}, \cos 3 \gamma, \cos ^{2} 3 \gamma$, and $\delta\left(\gamma-\gamma_{0}\right)$ :

$$
\begin{aligned}
& \left\langle n\left|p_{\gamma}\right| n^{\prime}\right\rangle=n \delta_{n, n^{\prime}} \\
& \left\langle n|\cos 3 \gamma| n^{\prime}\right\rangle=\frac{1}{2} \delta_{\left|n-n^{\prime}\right|, 3} \\
& \left\langle n\left|\cos ^{2} 3 \gamma\right| n^{\prime}\right\rangle=\frac{1}{2} \delta_{n, n^{\prime}}+\frac{1}{4} \delta_{\left|n-n^{\prime}\right|, 6} \\
& \left\langle n\left|\delta\left(\gamma-\gamma_{0}\right)\right| n^{\prime}\right\rangle=\exp \left[i\left(n^{\prime}-n\right) \gamma_{0}\right] / 2 \pi,
\end{aligned}
$$

where $\delta_{n, p}$ is the Kronecker delta.
The expectation value of $\delta\left(\gamma-\gamma_{0}\right)$ for a $J, K_{a}, K_{c}, M, v_{t}$ rotation-torsion levels belonging to the symmetry species $\Gamma$ is calculated as follows. When $\Gamma$ is a nondegenerate symmetry species:

$$
\left\langle\delta\left(\gamma-\gamma_{0}\right)\right\rangle=\left\langle\Psi^{\Gamma}\left|\delta\left(\gamma-\gamma_{0}\right)\right| \Psi^{\Gamma}\right\rangle
$$

When $\Gamma$ is a doubly nondegenerate symmetry species:

$$
\begin{aligned}
\left\langle\delta\left(\gamma-\gamma_{0}\right)\right\rangle= & \frac{1}{2}\left[\left\langle\Psi^{\Gamma_{a}}\left|\delta\left(\gamma-\gamma_{0}\right)\right| \Psi^{\Gamma_{a}}\right\rangle\right. \\
& \left.+\left\langle\Psi^{\Gamma_{b}}\left|\delta\left(\gamma-\gamma_{0}\right)\right| \Psi^{\Gamma_{b}}\right\rangle\right]
\end{aligned}
$$

which is independent on the way component functions are chosen. For both Eqs. (A2) and (A3), $\left|\Psi^{\Gamma}\right\rangle$ is a shorthand notation for the rotation-torsion wavefunction of Eq. (5), $\left|\Psi_{J K_{a} K_{c}, M, v_{t}}^{\Gamma}\right\rangle$.
[1] T. Muraoka, K. Kinbara, and T. Aida, Nature (London) 440, 512 (2006).

[2] I. Petreska, L. Pejov, and L. Kocarev, Phys. Rev. B 78, 045209 (2008).

[3] A. J. Merer and J. K. G. Watson, J. Mol. Spectrosc. 47, 499 (1973).

[4] S. Ramakrishna and T. Seideman, Phys. Rev. Lett. 99, 103001 (2007).

[5] C. B. Madsen, L. B. Madsen, S. S. Viftrup, M. P. Johansson, T. B. Poulsen, L. Holmegaard, V. Kumarappan, K. A. Jørgensen, and H. Stapelfeldt, J. Chem. Phys. 130, 234310 (2009).

[6] L. H. Coudert, L. F. Pacios, and J. Ortigoso, Phys. Rev. Lett. 107, 113004 (2011).

[7] J. Haruyama, C. Hu, and K. Watanabe, Phys. Rev. A 85, 062511 (2012).

[8] J. Floss, T. Grohmann, M. Leibscher, and T. Seideman, J. Chem. Phys. 136, 084309 (2012).

[9] J. Ortigoso and L. H. Coudert, Phys. Rev. A 87, 043403 (2013).

[10] T. Grohmann, M. Leibscher, and T. Seideman, Phys. Rev. Lett. 118, 203201 (2017).

[11] T. Grohmann, T. Seideman, and M. Leibscher, J. Chem. Phys. 148, 094304 (2018).

[12] C. B. Madsen, L. B. Madsen, S. S. Viftrup, M. P. Johansson, T. B. Poulsen, L. Holmegaard, V. Kumarappan, K. A. Jørgensen, and H. Stapelfeldt, Phys. Rev. Lett. 102, 073007 (2009).

[13] J. L. Hansen, J. H. Nielsen, C. B. Madsen, A. T. Lindhardt, M. P. Johansson, T. Skrydstrup, L. B. Madsen, and H. Stapelfeldt, J. Chem. Phys. 136, 204310 (2012).

[14] L. Christensen, J. H. Nielsen, C. B. Brandt, C. B. Madsen, L. B. Madsen, C. S. Slater, A. Lauer, M. Brouard, M. P. Johansson, B. Shepperson, and H. Stapelfeldt, Phys. Rev. Lett. 113, 073005 (2014).

[15] L. V. Thesing, A. Yachmenev, R. González-Férez, and J. Küpper, Phys. Rev. A 98, 053412 (2018).

[16] K. Hoki, D. Kröner, and J. Manz, Chem. Phys. 267, 59 (2001).
[17] S. M. Parker, M. A. Ratner, and T. Seideman, Mol. Phys. 110, 1941 (2012).

[18] G. Giraud, C. M. Gordon, I. R. Dunkin, and K. Wynne, J. Chem. Phys. 119, 464 (2003).

[19] H. Zhang, F. Billard, X. Yu, O. Faucher, and B. Lavorel, J. Chem. Phys. 148, 124303 (2018).

[20] J.-M. Hartmann and C. Boulet, J. Chem. Phys. 136, $184302(2012)$.

[21] L. H. Coudert and J. C. López, J. Mol. Spectrosc. 239, 135 (2006).

[22] M. Tudorie, L. H. Coudert, T. R. Huet, D. Jegouso, and G. Sedes, J. Chem. Phys. 134, 074314 (2011).

[23] J. T. Hougen, I. Kleiner, and M. Godefroid, J. Mol. Spectrosc. 163, 559 (1994).

[24] K. T. Hecht and D. M. Dennison, J. Chem. Phys. 26, 48 (1957).

[25] B. Kirtman, J. Chem. Phys. 37, 2516 (1962).

[26] R. M. Lees and J. G. Baker, J. Chem. Phys. 48, 5299 (1968).

[27] E. Herbst, J. K. Messer, F. C. De Lucia, and P. Helminger, J. Mol. Spectrosc. 108, 42 (1984).

[28] L.-H. Xu, J. Fisher, R. M. Lees, H. Y. Shi, J. T. Hougen, J. C. Pearson, B. J. Drouin, G. A. Blake, and R. Braakman, J. Mol. Spectrosc. 251, 305 (2008).

[29] E. P. Wigner, Group Theory (Academic Press, New York, 1959).

[30] Y. Y. Kwan and D. M. Dennison, J. Mol. Spectrosc. 43, 291 (1972).

[31] T. Seideman, J. Chem. Phys. 111, 4397 (1999).

[32] T. Seideman and E. Hamilton, in Advances In Atomic, Molecular, and Optical Physics, Vol. 52, edited by P. Berman and C. Lin (Academic Press, 2005) pp. 289329.

[33] N. Moiseyev and T. Seideman, J. Phys. B: Atomic, Molecular and Optical Physics 39, L211 (2006).

[34] S. L. Davis and E. C. Dennison, J. Chem. Phys. 101, 1293 (1994).

[35] A. R. Edmonds, Angular Momentum in Quantum Mechanics (Princeton University Press, Princeton, 1960). 
[36] L. H. Coudert, J. Chem. Phys. 148, 094306 (2018).

[37] S. Ramakrishna and T. Seideman, J. Chem. Phys. 124, 034101 (2006).

[38] B. A. Ashwell, S. Ramakrishna, and T. Seideman, J. Phys. Chem. C 117, 22391 (2013).

[39] C. C. Lin and J. D. Swalen, Rev. Mod. Phys. 31, 841 (1959).

[40] R. Escribano, B. Maté, F. Ortigoso, and J. Ortigoso, Phys. Rev. A 62, 023407 (2000).

[41] V. Renard, M. Renard, A. Rouzée, S. Guérin, H. R. Jauslin, B. Lavorel, and O. Faucher, Phys. Rev. A 70, 033420 (2004).

[42] L. H. Coudert, J. Chem. Phys. 146, 024303 (2017).

[43] H. Tal-Ezer and R. Kosloff, J. Chem. Phys. 81, 3967 (1984).

[44] C. Leforestier, R. H. Bisseling, C. Cerjan, M. D. Feit,
R. Friesner, A. Guldberg, A. Hammerich, G. Jolicard, W. Karrlein, H.-D. Meyer, N. Lipkin, O. Roncero, and R. Kosloff, J. Comput. Phys. 94, 59 (1991).

[45] A. Rouzée, S. Guérin, V. Boudon, B. Lavorel, and O. Faucher, Phys. Rev. A 73, 033418 (2006).

[46] G. Moruzzi, F. Strumia, C. Bonetti, B. Carli, F. Mencaraglia, M. Carlotti, G. Di Lonardo, and A. Trombetti, J. Mol. Spectrosc. 105, 24 (1984).

[47] L. Coudert and A. Valentin, J. Mol. Spectrosc. 122, 390 (1987).

[48] L. H. Coudert, Phys. Rev. A 91, 013402 (2015).

[49] H. Guo and R. Chen, J. Chem. Phys. 110, 6626 (1999).

[50] O. V. Boyarkin, T. R. Rizzo, and D. S. Perry, J. Chem. Phys. 110, 11346 (1999). 